\title{
Generalized hyperpigmentation after pyrimethamine use
}

\section{Ann Sterkens $s^{1,2}$, Vasiliki Siozopoulou ${ }^{1,2}$, Evelyne Mangodt ${ }^{1,2}$, Julien Lambert ${ }^{1,2}$, An Bervoets ${ }^{1,2}$}

${ }^{1}$ Faculty of Medicine, University of Antwerp, Wilrijk, Belgium, ${ }^{2}$ Dermatology Department, University Hospital Antwerp, Edegem, Belgium

Corresponding author: Dr. Ann Sterkens, E-mail: ann.sterkens@uza.be

\begin{abstract}
A 44-year old trans woman of Asian origin presented with generalized hyperpigmentation all over her body for four weeks. Six months before, she presented at the emergency department with motor ataxia and further investigation revealed a new diagnosis of HIV, cerebral toxoplasmosis with cerebral edema and latent syphilis. Two months later she presented with encephalitis: CMV and TBC were diagnosed. At that moment, a lot of new medication was started. Skin biopsy was compatible with a medical eruption. Hyperpigmentation was most probably caused by pyrimethamine, which was given for the treatment of toxoplasmosis. Pyrimethamine was stopped and changed into trimethoprim and sulfamethoxazole. Reevaluation after two months showed fading of the hyperpigmentation, especially in the face, which points towards the right diagnosis.
\end{abstract}

Key words: Generalized hyperpigmentation; Toxoplasmosis; Pyrimethamine; HIV, Trimethoprim; Sulfamethoxazole

\section{INTRODUCTION}

Hyperpigmentation is the darkening of the skin. Most of the time it is caused by an increased melanin deposition, and in rare cases it is due to deposition of pigments like iron or hemosiderin. Hyperpigmentation can be well circumscribed (lentigines, ephilides, maturational hyperpigmentation, melasma, fixed drug eruption,...) or diffuse. The diffuse pattern can be linear (phytophotodermatitis, drug-induced), reticulated (drug-induced, erythema ab igne, confluent papillomatosis), nonpatterned (drug-induced, idiopathic) or it can be associated with an endocrine/ metabolic or auto-immune disease.

Normally melanin can only be found in the epidermis. Melanin in the dermis can occur in inflammatory skin diseases, ectopic melanocytes and binding of melanin to exogenous pigments in the dermis. Wood's light examination can be useful to determinate where most of the pigment is located, but it is not useful in dark skinned patients. Skin biopsy is not a routine examination but can be done when the clinical diagnosis is not clear.

\section{CASE REPORT}

A 44-year old transwoman of Asian origin, who recently came to Belgium, presented with generalized hyperpigmentation all over the body, also in sunprotected areas (Figs la and lb). It started four weeks before with xerosis and flaking of the skin and it evolved to hyperpigmentation. Less hyperpigmentation was visible at the nose bridge and in the infra-orbital region.

She had recently been suffering from numerous medical problems.

Six months ago, she went to the emergency room because of motor ataxia of the right hand and leg, due to cerebral edema. A blood test revealed a new diagnosis of HIV (CD4 20 cells/ $\mu \mathrm{l}$ ) and latent syphilis.

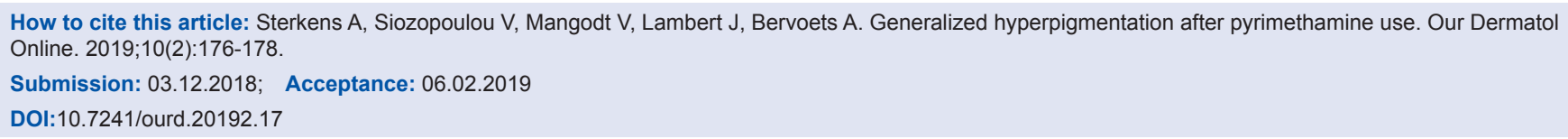




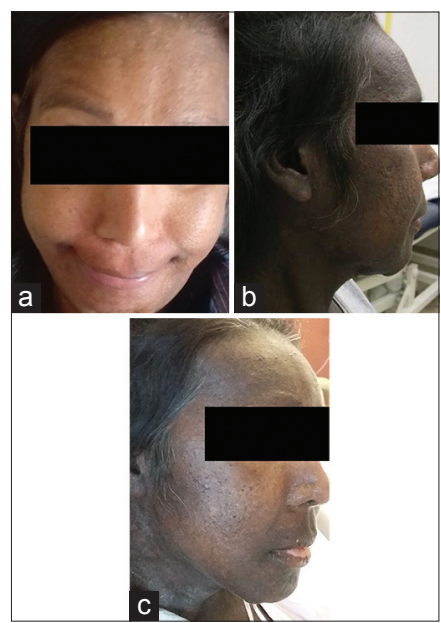

Figure 1: (a) Face before hyperpigmentation (b) Hyperpigmented face at the moment of diagnosis (c)Two months after stopping pyrimethamine: hyperpigmentation of the face starts to fade away.

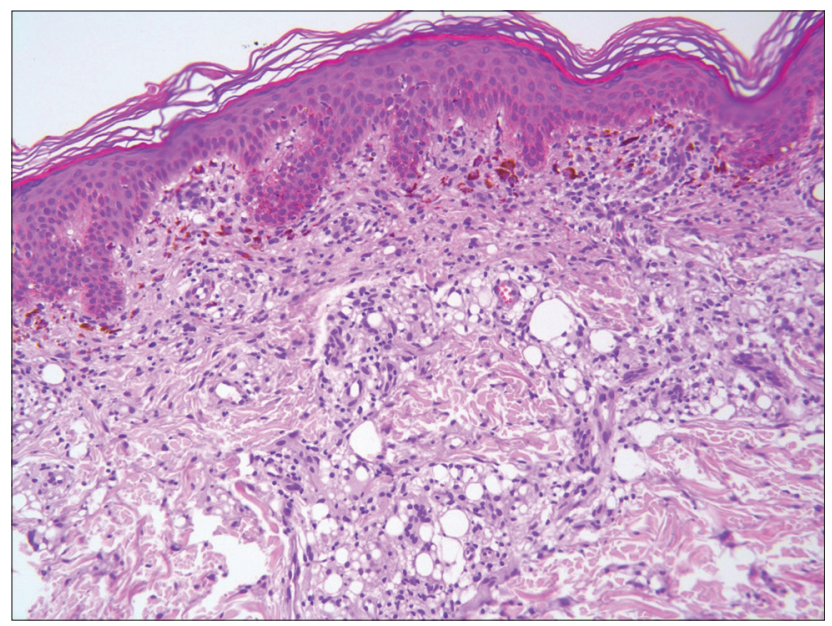

Figure 2: Skin biopsy: melanin incontinence.

A sample of cerebrospinal fluid (CSF) confirmed the diagnosis of cerebral toxoplasmosis. Highly Active AntiRetroviral Therapy (HAART) was started (dolutegravir 200/245 mg lx/day and emtricitabine/tenofovir disoproxil $50 \mathrm{mg} \mathrm{lx} /$ day) and toxoplasmosis was treated with pyrimethamine $25 \mathrm{mg} 3 \mathrm{x} /$ day, sulfadiazine 4x/day, levofolic acid $15 \mathrm{mg} 1 \mathrm{x} /$ day and folic acid. Dexamethasone $4 \mathrm{mg} / \mathrm{dl}$ 6x/day, was given to treat cerebral edema and penicillin 1200000 IE for syphilis.

Four months ago, she presented with an evolutive sepsis and encephalopathy. Bilateral retinitis and pneumonitis, caused by cytomegalovirus (CMV), were diagnosed and treated with valganciclovir $450 \mathrm{mg} \mathrm{2x1/}$ day. X-ray and CT of the thorax were suggestive for TBC pneumonitis but PCR and Ziehl stain were negative. However, anamnesis and imaging were very suspicious for TBC, tuberculostatic medication was started. Pyrazinamide $500 \mathrm{mg} \mathrm{lx4/day,} \mathrm{isoniazid} 300 \mathrm{mg} \mathrm{lxl/}$ day, ethambutol $400 \mathrm{mg}$ 1x1/day and rifampicin 1x2/ day were started.

For a male to female transition she was treated with a feminizing hormone therapy (estradiol $2 \mathrm{mg} \mathrm{lx}$ /day and cyproterone $50 \mathrm{mg} \mathrm{lx} /$ day), but this medication had already been stopped a few months before she presented with hyperpigmentation. She also used isotretinoïn $(20 \mathrm{mg} \mathrm{lx} / \mathrm{d})$ for the treatment of acne.

A skin biopsy was taken and showed interphase pathology with melanin incontinence, compatible with a drug eruption (Fig. 2).

Prior to the study, patient gave consent to the examination and biopsy after having been informed about the procedure.

\section{DISCUSSION}

HIV positive patients suffer from a lot of dermatological manifestations. Most frequent problems are dry skin, seborrheic dermatitis, Kaposi sarcoma, pruritus and oral candidiasis. Sometimes these problems are the first symptoms of HIV, whereby further research leads to the diagnosis of HIV. The rate of skin manifestations is linked to disease progression. Oral candidiasis and Kaposi's sarcoma are significantly correlated with low CD4+ counts $[1,2]$.

This patient had a non-patterned generalized hyperpigmentation of the skin. Most likely, the hyperpigmentation is drug-induced. Normally melanin can only be found in the epidermis. Is this case, discoloration of skin and mucosa is caused by an increased melanin production or a deposition of drug complexes in the dermis. Wood's light examination can be useful to determinate whether most of the pigment is located at the epidermis or dermis, but it is not useful in dark skinned patients [3].

Chemotherapeutic agents, antimalarials, hormones, heavy metals, prostaglandin agonist, smoking and some others (Zivoduvine, Minocycline, Psoralens, Amiodaron, Clofazimine..,) can be responsible [4,5].

This patient took a lot of medication. Pyrazinamide, co-trimoxazole and esomeprazole are known to cause photosensitivity. In this case, photosensitivity or phototoxicity as an etiology of the hyperpigmentation is unlikely because sun-protected areas are also discolored. 
Emcitrabine is known to cause hyperpigmentation in children, but it is not yet described in literature for adults [6].

A rare side-effect of pyrimethamine is abnormal skin pigmentation.

In 2011, a case report has been published which described a generalized hyperpigmentation in a 7-year old girl. The girl was diagnosed with toxoplasmosis and treated with a loading dose of $40 \mathrm{mg}$ pyrimethamine, followed by $20 \mathrm{mg}$ pyrimethamine a day. Medication was changed and two months later the hyperpigmentation started to fade away [7].

Pyrimethamine is the most likely cause of hyperpigmentation in this woman. Medication was stopped and changed into trimethoprim and sulfamethoxazole. Two months later, discoloration started to fade away, especially in the face (Fig. 1c).

\section{CONCLUSION}

There is not yet much published about pyrimethamine and discoloration. In our case pyrimethamine is most likely the responsible drug because after stopping the medication, hyperpigmentation started to fade away which points toward the right diagnosis.

\section{Consent}

The examination of the patient was conducted according to the Declaration of Helsinki principles.

\section{REFERENCES}

1 Chawhan SM, Bhat DM, Solanke SM. Dermatological manifestations in human immunodeficiency virus infected patients: Morphological spectrum with CD4 correlation. Indian J Sex Trans Dis AIDS. 2013;34:89-94.

2. Singh H, Singh P, Tiwari P, Dey V, Dulhani N, Singh A. Dermatological manifestations in HIV-infected patients at a tertiary care hospital in a tribal (bastar) region of Chhattisgarh, India. Indian J Dermatol. 2009;54:338-41.

3. Manjunath K, Kiran C, Sonakshi S, Ashwini N, Agrawal R. Comparative study of Woods lamp and dermoscopic features of melasma. J Eviden Bas Med Healthcare. 2015;2:9012-15.

4. Ghosh A, Das A, Sarkar R. Diffuse hyperpigmentation: A comprehensive approach. Pigment Int. 2018;5:4-13

5. Dessirier F, Arnault J-P, Farre I, Poulet C, Chaby G, Dairi M, et al. Atypical cutaneous hyperpigmentation after multiple chemotherapy agents. Our Dermatol Online. 2017;8:276-9.

6. Sennayake MP, Mettananda DSG, Vidyatilake S. Profound trombocytopenia and generalized hyperpigmentation following pyrimethamine use. Sri Lanka J Child Health. 2011,40:134-5.

7. Engin A, Ozaras R, Mert A, Tabak F, Aktuğlu Y. Hyperpigmentation due to pyrimethamine use. J Dermatol. 2002;29:443-5.

Copyright by Ann Sterkens, et al. This is an open-access article distributed under the terms of the Creative Commons Attribution License, which permits unrestricted use, distribution, and reproduction in any medium, provided the original author and source are credited.

Source of Support: Nil, Conflict of Interest: None declared. 